\title{
Fracture mechanism of magnesium alloys at room temperature
}

\author{
Shan Jiang ${ }^{\text {a }}$ \\ Research Institute for New Materials and Technology, Chongqing University of Arts and \\ Sciences, No. 329, Hong he Road, Yongchuan District 402160, China \\ a382595277@qq.com
}

Keywords: Magnesium alloys, Deformation, Slip, Twinning, Grain boundary sliding.

\begin{abstract}
Compressive tests were performed to homogenized AZ31 magnesium alloy samples to various strains and fracture at room temperature (RT). The microstructure of the samples was observed by optical microscope(OM); the twin types were examined by electron backscattered diffraction (EBSD) technique; and the fracture surfaces were detected by scanning electron microscope (SEM). The fracture mechanism was discussed by considering the interaction among basal slip, twinning and interface motion. It was considered that basal slip and $\{10 \overline{1} 2\}$ twinning work together during deformation. Basal slip may result in the potential origin of fracture at grain boundaries (GBs); while the interface of $\{10 \overline{1} 2\}$ twins may become the favorable source of fracture across the grains.
\end{abstract}

\section{Introduction}

The fracture behaviors have a crucial impact on the material properties [1-4], therefore the researches on the fracture mechanism are of great importance for the optimization of material forming processing. Though many studies have been conducted on the deformation of $\mathrm{Mg}$ alloys [5-7] origin of their fracture properties has not yet been well understood. Scientifically, an emerging consensus is that the poor processing behavior of $\mathrm{Mg}$ alloys is due to their hexagonal crystal structure (HCP), where slips upon their non-basal systems have a remarkably large critical resolved shear stress (CRSS) that refrains them from being activated at RT and hence from satisfying the five slip systems required by the Von Mises law. In general, it is believed that the deformation behavior of $\mathrm{Mg}$ alloys is governed by deformation twinning and basal slip at RT [8]. One non-negligible issue in the deformation mechanism of Mg alloys may rests with the role of GBs, because the block of them will directly lead to the fracture of the materials [9-11]. Conversely, both twinning and basal slip can induce the motion of GBs [12], but in rather different manners: the effect of the former is much less significant than that of the latter owing to the limited deformation that twinning shear can contribute. For this reason, we hereby focus mainly on the relationship among basal slip, twinning and GB motion.

\section{Experimental procedures}

The material used was commercial AZ31 Mg alloy with chemical composition (in mass \%) of $2.9 \% \mathrm{Al}, 0.98 \% \mathrm{Zn}, 0.29 \% \mathrm{Mn}$, and $\mathrm{Mg}$ (in balance), which was provided in form of cast ingots. The ingots were first machined to cuboid samples with dimension of $20 \times 20 \times 40 \mathrm{~mm}^{3}$ using the wire cutting technology, and then homogenized at $723 \mathrm{~K}$ for $5 \mathrm{~h}$ in order to eliminate the dendrites. Compression tests were conducted parallel to the long axis of the samples under an identical rate of $0.001 \mathrm{~s}^{-1}$ at RT using the universal material testing machine (CMT25150). Microstructures were observed using OM. The specimens for OM observation were prepared by grinding and polishing firstly, and followed by a corrosion in a etching solution composed chemically of $5 \mathrm{~g}$ picric acid, 5 g acetic acid, $10 \mathrm{~mL}$ distilled water, and $100 \mathrm{~mL}$ ethanol. The SEM detection were performed using Noval 400 Nano SEM under $20 \mathrm{kV}$ with a working distance of $20 \mathrm{~mm}$ and a tilt angle of $70^{\circ}$. 


\section{Results}

Figure 1 presents a typical stress-strain curve for the homogenized sample compressed at RT characterized as a steady increase of stress with rise of strain. The ultimate compressive strength (UCS) is estimated to be about $250 \mathrm{MPa}$, and failure occurs at the strain of approximately $28 \%$.

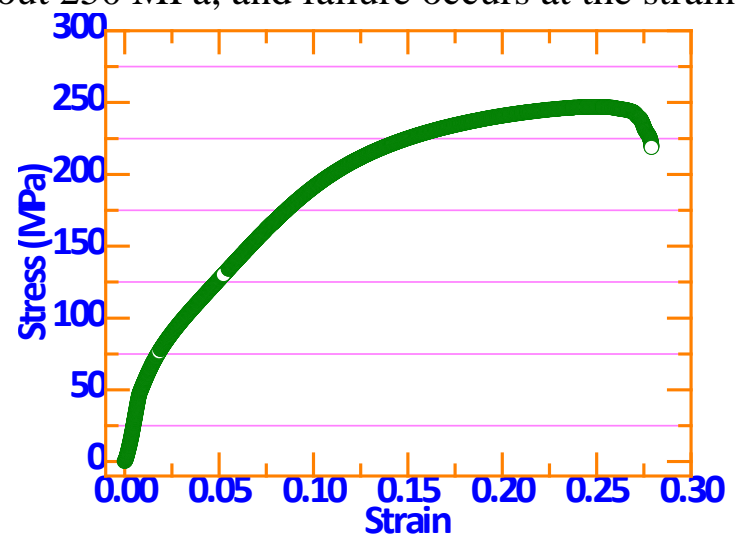

Fig. 1 RT compression flow curve of an AZ31 alloy sample homogenized at $723 \mathrm{~K}$ for $5 \mathrm{~h}$.

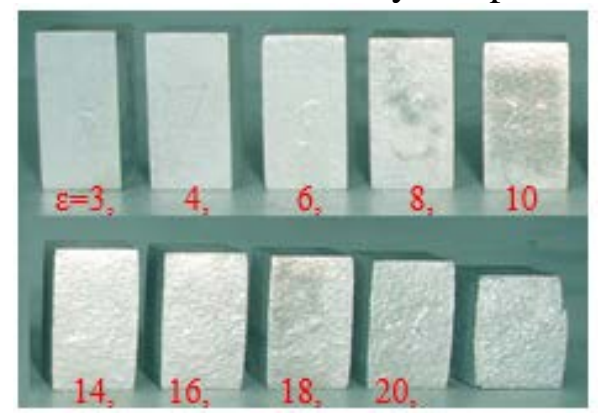

Fig. 2 AZ31 alloy samples compressed to various strains and fracture at RT.

Figure 2 shows the samples compressed to different strains and fracture, in which. The surface of the fracture sample is at about an angle of $45^{\circ}$ to the compressive axis. Figure 3 shows the metallographic microstructures of the AZ31 alloys deformed to the strains of $0.05,0.1$ and 0.15 , where the twins with diverse morphologies can be observed. For the sample to the strain of 0.05 , the deformation induces the formation of secondary twins and crossing twins (Fig. 3a). As deformation proceeds, the amount of twins increases noticeably and some twins start to turn wider (Fig. 3b). Evidently, the twins formed at a later stage are blocked by those generated earlier. At the final stage, twins fill in most area of grains and become saturated (Fig. 3c), and the twin intersection can be noticeably observed, which demonstrates that the movement of twinning is intensified at his stage. Figure 3(d) indicates that most of the twins formed are $\{1012\}$ type (misoriented by about $86^{\circ}$ ), and only a few of them are the $\{10 \overline{1} 1\}$ type (misoriented by about $56^{\circ}$ ) and $\{10 \overline{1} 2\}-\{10 \overline{1} 1\}$ double type (misoriented by about $38^{\circ}$ ).

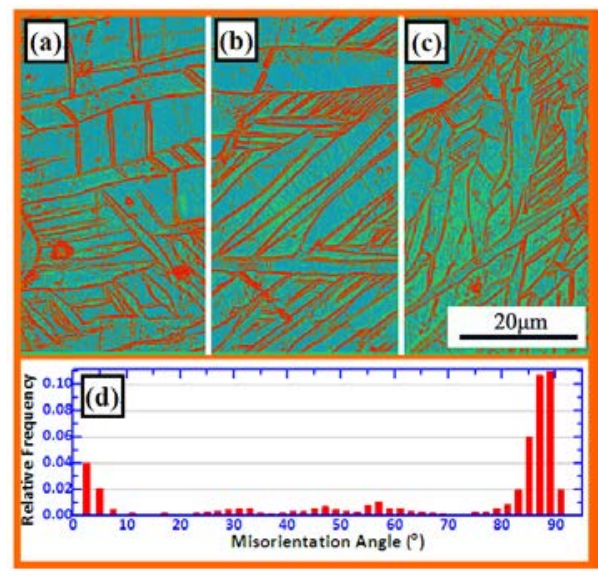

Fig. 3 Optical micrographs of the AZ31 alloy samples compressed to various strains (a) 0.05; (b) 
0.1 ; (c) 0.15 and (d) relation between the relative frequency and the disorientation angle in the sample of 0.1 strain.

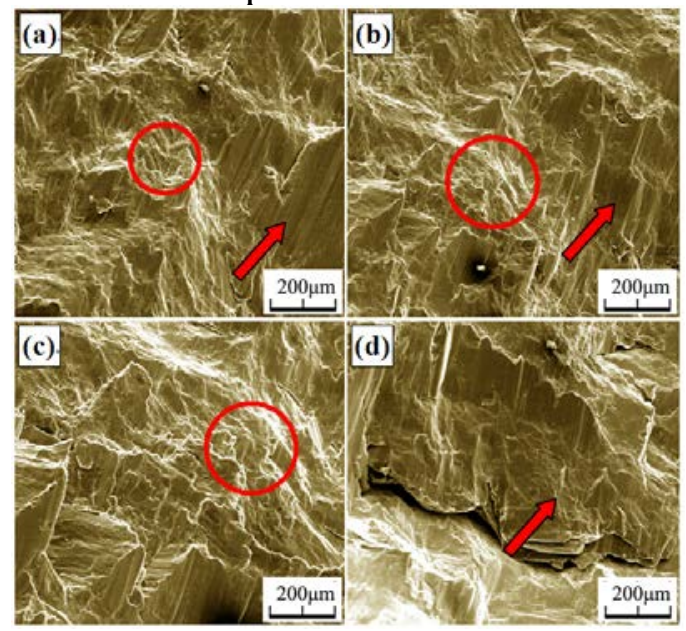

Fig. 4 Schematic diagram of two adjacent asymmetric grains before (a) and after (b) GB sliding.

As shown in Fig. 4, the fracture surface of the AZ31 magnesium alloy is a typical brittle cleavage fracture (indicated by the arrows) where also appears some cleavage steps (indicated by the circles).

\section{Discussions}

As shown in Fig. 5, when the basal planes are at an angle of $45^{\circ}$ to the compressive direction, basal slip is inclined to be activated in the grain (Fig. 5a); when the basal planes are perpendicular to the compressive direction (Fig. 5b), neither basal slip nor twinning will occur; and when the basal planes are parallel to the compressive direction, $\{10 \overline{1} 2\}$ twinning is likely occurs (Fig. 5c).

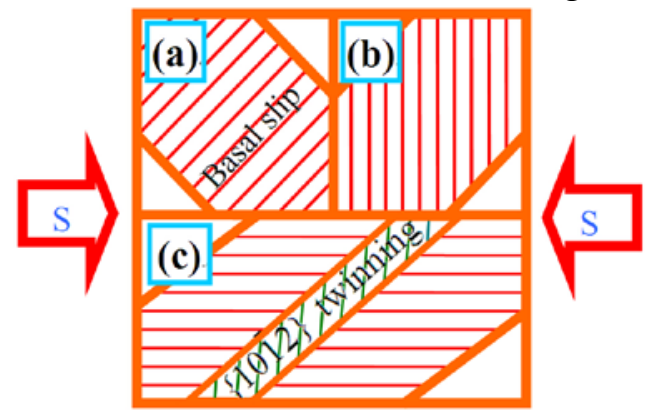

Fig. 5 Inclined deformation mechanism of the grains with different orientations under compressive stress at RT: (a) Basal slip; (b) neither basal slip nor $\{10 \overline{1} 2\}$ twinning; and (c) $\{10 \overline{1} 2\}$ twining.

\section{1 Influence of basal slip on deformation}

Here, the influence of basal slip to the grain boundary sliding will be discussed. In view of the fact that GBs are in a complicated environment, we addressed a usual case to demonstrate how basal slip affects GB motion: GBs are assumed to be between two randomly oriented grains with no external influence (Fig. 6). In addition, the resistance between grains at the boundary is supposed to be small enough to let the GB sliding can be activated under certain stress. 

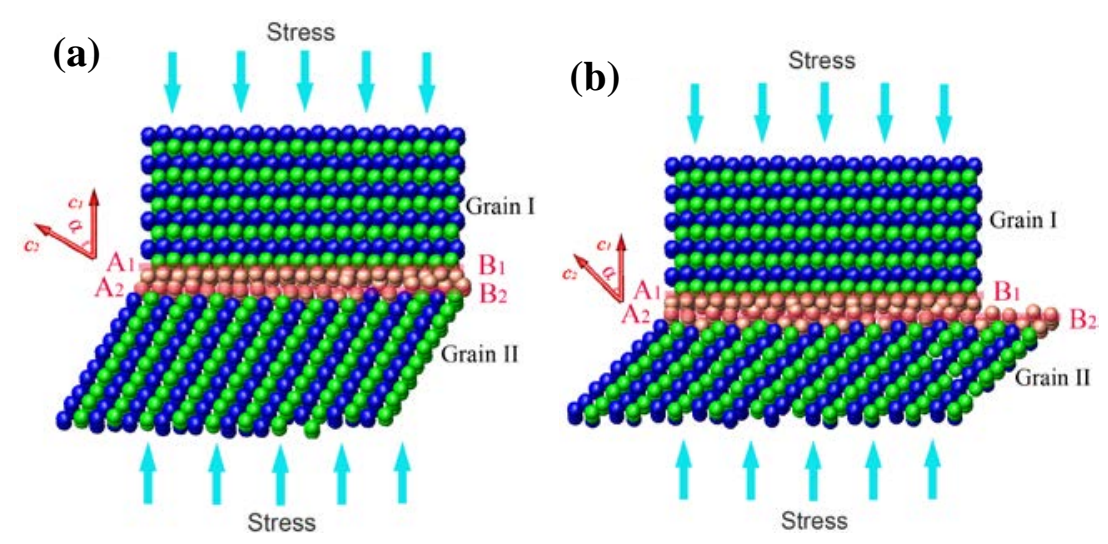

Fig. 6 Schematic diagram of two adjacent asymmetric grains. (a) Before and (b) after basal slip.

Since whether a basal slip can be activated is closely related to grain orientation, we expressed the relationship between critical resolved shear stress (CRSS) $\sigma_{\mathrm{s}}$ and the yield stress $\tau_{\mathrm{c}}$ in a single crystal as follows:
$\tau_{\mathrm{c}}=\sigma_{\mathrm{s}} \cos \varphi \cos \lambda$
$m=\cos \varphi \cos \lambda$,

Where $\varphi$ and $\lambda$ are the angles between tensile (compress) axis and c-axis, and between tensile (compress) axis and the basal planes, respectively; and $m$ is the Schmid factor (or orientation factor). In light of the Eq. (1) and (2), when two oriented grains I and II are under a compressive stress (Fig. 6), basal slip in the grain I are difficult to be initiated due to their hard orientations; comparatively, basal slip in the grain II is more preferable to be activated due to its soft orientations. As can be seen from Fig. 6, the grain boundary can be clearly divided into two parts: the upper part $A_{1} B_{1}$ and the lower part $A_{2} B_{2}$ (Fig. 6a). If the $A_{1} B_{1}$ keeps unchanged, the $A_{2} B_{2}$ in the grain II must be elongated as the basal slip proceeds (Fig. 6b). In this sense, magnitude of the GB sliding can be defined as the length of $\mathrm{B}_{1} \mathrm{~B}_{2}$ between the two grains.

We therefore conclude that basal slip, GB sliding and grain rotation always concur, forcing the basal planes to rotate and make their orientations approach to the textures. This conclusion is essential for a further study on the fracture in Mg alloys because the origin of the stress in twin boundaries before fracture remain unclear, although a consensus on the occurrence of fracture along the twin boundaries during room-temperature compression has been reached. Further, these analyses reveal that the stress accumulation is responsible for the fracture originates from the basal slip at the final stage of deformation. When a basal is activated by stress, the orientation of basal planes in grains is modulated to approach the normal of the stress direction, which thereby induces GB motion or fracture.

\section{2 Influence of $\{10 \overline{1} 2\}$ twinning on deformation}

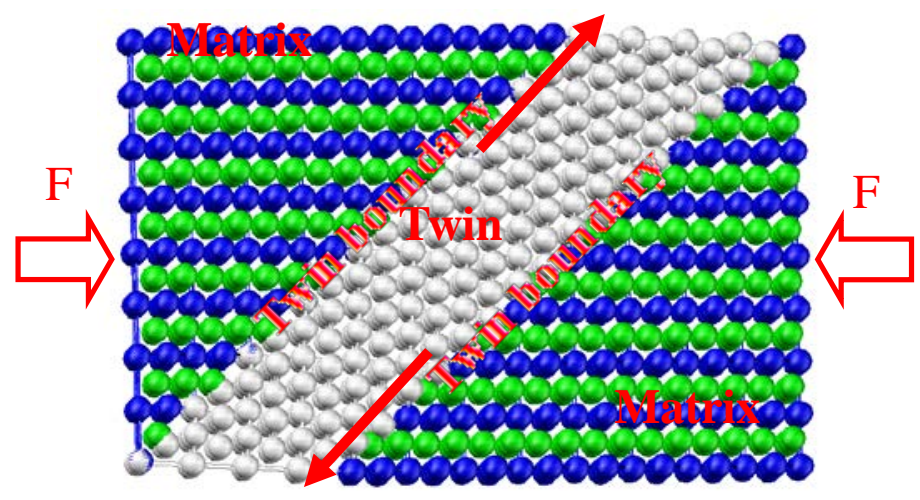

Fig. 7 Schematic diagram of the tendency of twin boundary motion under stress.

Under compression, grains with their $c$-axes perpendicular to the compressive direction are oriented favorably for twinning till the $c$-axes of the inner twins becomes almost aligned with the 
compression axis. For Mg alloys with the axis ratio (c/a) of about 1.623 (here $a$ and $c$ are lattice constants of hexagonal lattice), the $\{10 \overline{1} 2\}$ twinning is generally inclined to allow additional extensions along the $c$-axis of hexagonal lattice. However, because the shear that $\{10 \overline{1} 2\}$ twinning can supply is rather low (only about 0.13 ), the theoretical contribution of the twinning alone to deformation (i.e., enhancement of total plasticity) has been found to be rather limited (about only 6\%). Although twinning can shift orientation of grains abruptly from that of their basal planes [13], it is difficult to reactivate other slip systems within the twins for that twin boundaries can act as the barriers for the dislocation motion [14].

As mentioned above, the initiation of basal slip will induce stress concentration at the grain boundaries, which may produce grain boundary sliding or lead to the fracture at the boundaries. When the twins reach saturation in the grains and the twin boundaries are blocked, the activation of basal slip within the twins will exert shear stress to the twin boundaries and induce the cracks source there; in addition, because the twin boundaries are rather smooth compared with the common grain boundaries, once the cracks source appears, it will expand rapidly and result in materials fracture.

\section{Summary}

1) For the compressive deformation of magnesium alloys, the grains can be divided into three species according to their orientations: with their $c$-axes parallel, at an angle of $45^{\circ}$ and perpendicular to the compressive axis, in which the corresponding inclined deformation mechanisms are $\{10 \overline{1} 2\}$ twinning, basal slip and neither of the two.

2) The fracture mode of Magnesium alloy compressive deformation belongs to brittle cleavage type, the surface of which is at an angle of about $45^{\circ}$ to the compression direction, which indicates that when $\{10 \overline{1} 2\}$ twins fill out the grains, the blocked twin boundaries at an angle of $45^{\circ}$ to the compression direction become the favorable source of fracture.

3) Basal slip plays a major role in the deformation for that the actual deformation of material (0.28) is much larger than the theoretical contribution of $\{10 \overline{1} 2\}$ twinning to the deformation (approximately only 0.06), and it will eventually lead to GB sliding or fracture at GBs.

\section{Acknowledgements}

This work is supported by National Natural Science Foundation of China (No. 51301215), Natural Science Foundation Project of Chongqing (No. cstc2012jjA 50016), Talent projects of Chongqing University of Arts and Sciences (R2014CJ04; Y2014CJ29; R2012CJ19), and Chongqing Post-doctor Support Program (Rc201334).

\section{References}

[1] B. Langelier, A. M. Nasiri, S. Y. Lee, et al. Improving microstructure and ductility in the Mg-Zn alloy system by combinational Ce-Ca microalloying. Mater. Sci. Eng. A. 620 (2015) 3, 76-84.

[2] H. Kwon, H. Nakano, M. Mabuchi, Y. Chino: Philos. Mag. 94 (2014) 3960-3977.

[3] W. Liu, L. Jiang, L. Cao. Fatigue behavior and plane-strain fracture toughness of sand-cast Mg-10Gd-3Y-0.5Zr magnesium alloy. Mater. Des. 59 (2014) 466-474.

[4] D. Ando, J. Koike, Y. Sutou. The role of deformation twinning in the fracture behavior and mechanism of basal textured magnesium alloys. Mater. Sci. Eng. A. 600 (2014) 10, 145-152.

[5] A. Singh, H. Somekawa, T. Mukai. Formation of nano-twin domains by nucleation and multiplication of twins during fracture of a magnesium alloy. Philos. Mag. 94 (2014) 9, 898-913.

[6] F. Feng, S. Huang, Z. Meng. A constitutive and fracture model for AZ31B magnesium alloy in the tensile state. Mater Sci Eng A. 594 (2014) 31, 334-343. 
[7] V. Kaushik, R. Narasimhan, R. K. Mishra. Experimental study of fracture behavior of magnesium single crystals. Mater. Sci. Eng. A. 590 (2014) 10, 174-185.

[8] H. Somekawa, T. Inoue, K. Tsuzaki. Effect of solute atoms on fracture toughness in dilute magnesium alloys. Philosl Mag. 93 (2013) 36, 4582-4592.

[9] G. Proust, C. N. Tomé, A. Jain, et al. Modeling the effect of twinning and detwinning during strain-path changes of magnesium alloy AZ3. Inter. J. Plasticity. 25 (2009) 5, 861-880.

[10] Y. Wang, J. Huang. Comparison of grain boundary sliding in fine grained $\mathrm{Mg}$ and $\mathrm{Al}$ alloys during superplastic deformation. Scripta Mater. 48 (2003) 14, 1117-1122.

[11]Y. Wang. J. Huang. The role of twinning and untwinning in yielding behavior in hot-extruded Mg-Al-Zn alloy. Acta Mater. 55 (2007) 3, 897-905.

[12]T. Al-Samman, X. Li, S. G. Chowdhury. Orientation dependent slip and twinning during compression and tension of strongly textured magnesium AZ31 alloy. Mater. Sci. Eng. A. 527 (2010) 15, 3450-3463.

[13]B. Clausen, C. N. Tome, D. W. Brown, et al. Reorientation and stress relaxation due to twinning: Modeling and experimental characterization for Mg. Acta Mater. 56 (2008) 11, 2456-2468.

[14]X. Lou, M. Li, R. K. Boger, et al. Hardening evolution of AZ31B Mg sheet. Int. J. Plasticity. 23 (2007) 1, 44-86. 